\title{
WAVELET REGULARITY OF ITERATED FILTER BANKS WITH RATIONAL SAMPLING CHANGES
}

\author{
Thierry Blu and Olivier Rioul \\ France Télécom, CNET Paris B \\ CRPE (Unité mixte CNRS/CNET) \\ 38-40 rue du Général Leclerc \\ 92131 Issy-Les-Moulineaux, France.
}

\begin{abstract}
The regularity property was first introduced by wavelet theory for octave-band "dyadic" filter banks. In this paper, we provide a detailed theoretical analysis of the regularity property in the more flexible case of filters banks with rational sampling changes. Such filter banks provide a finer analysis on fractions of an octave, and regularity is equally important as in the dyadic case. Sharp regularity estimates for any filter bank are given.

The major difficulty of the rational case, as compared to the dyadic case, is that one obtains "wavelets" that are not shifted versions of each other at a given scale. We show, however, that under regularity conditions, shift invariance can be almost obtained. This is a desirable property for e.g. coding applications and for efficient filter bank implementation of a continuous wavelet transform.
\end{abstract}

\section{INTRODUCTION}

Dyadic wavelet transforms, associated to octave-band FIR filter banks with sampling changes by two, have been successfully applied for applications such as subband coding of speech and images [10]. The regularity property was first introduced in this context by Daubechies [3] and was found to be a promising filter criterion for some applications [10]. It is the major novelty brought by wavelet theory over filter banks. Now, the dyadic case can be extended to include rational sampling rates, resulting in more flexible decomposition schemes: In the "rational" case, the signal is analyzed by fractions $\log _{2}(p / q)$ th of an octave instead of being analyzed octave by octave, resulting in a finer analysis if $1<p / q<2$. The dyadic case is, of course, recovered by setting $p / q=2$. Within this generalized framework, regularity of "wavelets" underlying rational filter banks should be equally important as in the well-known dyadic case.

Therefore, we consider the two-band rational filter bank as depicted in Fig. 1, where $G(z)$ and $H(z)$ are low-pass and high-pass filters, respectively. This filter bank is iterated over low-pass branches. Roughly speaking, wavelets are obtained as combinations of equivalent impulse responses of the low-pass branch, when it is iterated to infinity. The regularity problem is to find the conditions on low-pass filter $G(z)$ such that the corresponding wavelets are "regular," i.e., continuous, possibly with several continuous derivatives. Such regularity conditions will generate "smooth"

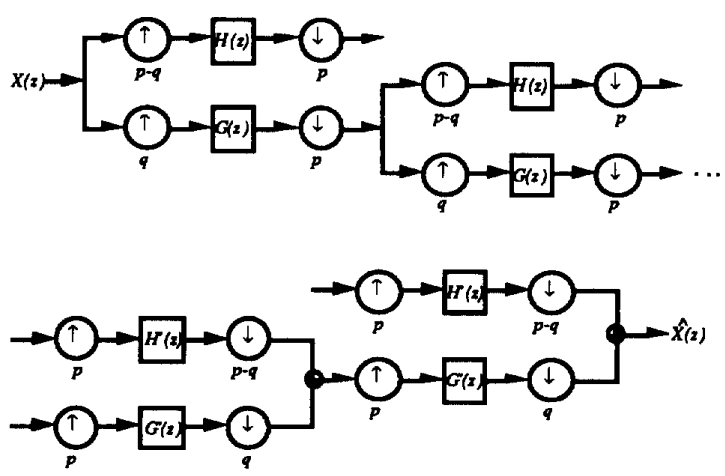

Figure 1: Flow-graph of iterated two-band rational filter bank.

impulse responses in the filter bank.

Kovačević and Vetterli [6] designed perfect reconstruction FIR filter banks with rational sampling rates, and were the first who investigated the existence of associated rational wavelets as limit functions. They first thought that regular rational wavelets could be obtained, and even gave an explicit example in [6]. However, they finally concluded [5], using a remark of Cohen and Daubechies [2], that it is theoretically impossible to construct such wavelets. The theoretical analysis undertaken in this paper explains why they were at first led to a positive conclusion, while also pointing out the existence of rational "pseudo-wavelets".

In fact, iterating $j$ times the low-pass branch gives the flow-graph of Fig. 2, where $G^{j}(z)$ is defined by

$$
G^{j}(z)=G\left(z^{q^{j-1}}\right) G\left(z^{p q^{j-2}}\right) \cdots G\left(z^{p^{j-2} q}\right) G\left(z^{p^{j-1}}\right)
$$

Kovačević and Vetterli [6] took the associated sequence $g_{n}^{j}$ as a candidate for converging to a limit function. But it turns out that this is impossible [5] for FIR filters.

There is a good reason for this impossibility: The impulse responses of Fig. 2 are, in fact, the decimated sequences $g_{n}^{j, s}=g_{q^{j} n-p^{j} s^{j}}^{j}$ and for each value of $s$ (shift parameter), we can now define a limit function $\varphi^{s}(t)$, as the limit as $j \rightarrow \infty$ of the discrete curves $g_{n}^{j, s}$ plotted against $n(p / q)^{-j}[1]$. The functions $\varphi^{s}(t)$ are the counterparts of

III-213

0-7803-0946-4/93 $\$ 3.00 \stackrel{\circ}{ } 1993$ IEEE 


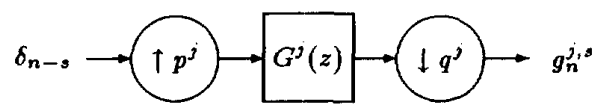

Figure 2: Flow-graph of the iterated low-pass branch, giving $g_{n}^{j, s}=g_{q^{j} n-p^{j} s}^{j}$.

the "scaling function" in a classical dyadic wavelet transform [3], and yield "pseudo-wavelets". In the dyadic case, since $g_{n}^{j, s}=g_{n-2 j_{s}}^{j}(p=2, q=1)$, one has $\varphi^{s}(t)=\varphi^{0}(t-s)$ : shift invariance is satisfied. Unfortunately, this shift invariance does not hold anymore whenever $q>1$, as illustrated in Fig. 3 (b). In other words, the shape of the equivalent impulse responses of rational FIR filter banks vary in time, resulting in infinitely many "wavelet prototypes".

We therefore have to consider an infinite set of limit functions rather than one, and study regularity of each of these functions. Fortunately, a theoretical analysis of their global regularity is possible and is undertaken in section 2 . It allows one to obtain algorithms which compute sharp regularity estimates for all limit functions $\varphi^{s}(t)$, given in section 3 . This is a necessary prerequisite for investigating the precise role of regularity for a particular application.

Regularity is useful not only for obtaining smooth impulse responses in an iterated rational filter bank, but also for obtaining shift invariance within a small error. We show in section 4 that rational pseudo-wavelets can be easily made almost "shiftable": Shift invariance can be obtained within a small error, precisely by increasing the regularity of these pseudo-wavelets.

\section{THEORETICAL ANALYSIS OF REGULARITY}

Regularity of limit functions $\varphi^{s}(t)$ represents the amount of "smoothness" that can be imposed by a suitable choice of low-pass filters $G(z)$ (in the analysis part) or $G^{\prime}(z)$ (in the synthesis part, see Fig. 1). Since wavelets are linear combinations of the $\varphi^{s}(t)$ [1], they are at least as regular. Under special conditions on the filters, the "wavelets" are regular as shown in Fig. 3 (a). For "bad" choices of the low-pass filter $G(z)$, however, they do not have smooth time evolutions (see each curve in Fig. 3 (b)). The aim of this section is to find these regularity conditions on $G(z)$.

Let us first note that "spectrum-based" methods, which have been successfully applied for estimating regularity in the dyadic case (see e.g. [3]) are no longer useful in the rational case, due to the lack of shift invariance. The only method we know that can be successfully generalized from the dyadic case to the rational one is the "discrete-time approach" investigated in $[7,8]$.

In the following, we outline the basic steps of this approach in the rational case. This development follows [8], and can be skipped for readers willing to avoid the technical details leading to the main results of this paper. We first need some intermediate results on derivatives and $N$ th order derivatives ((2) and (3)) and then use Hölder regularity (4) to arrive at the main discrete criterion (5), which serves as the basis for estimating regularity.
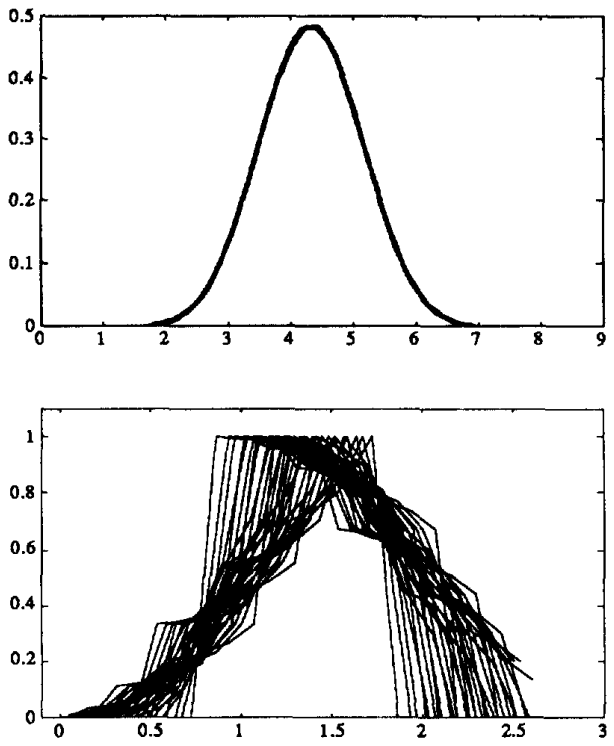

Figure 3: Plots of the limit functions $\varphi^{s}(t+s)$, for 32 different values of $s$. They were re-shifted to emphasize the shape similarities $(p / q=3 / 2)$. (a). Regular exemple $G(z)=\left(1+z^{-1}+z^{-2}\right)^{5}$, for which the shift invariance almost holds. (b). Continuous, but not very regular exemple, $G(z)=\left(1+z^{-2}\right)\left(1+z^{-1}+z^{-2}\right)$.

First, we characterize "uniform" convergence of $g_{n}^{j, s}$ to limit functions $\varphi^{s}(t)$ - which are then all continuous-by the fact that the maximum of the differences $\left|g_{n}^{j, s}-g_{n-1}^{j, s}\right|$ tend to 0 as $j \rightarrow \infty$. Intuitively speaking, no discontinuities occur in the iteration process, hence the limit functions are continuous. Moreover, this implies that at least one factor of the form $\frac{1-z^{-p}}{1-z^{-1}}$ divides $G(z)[1]$.

However, continuity is not enough to ensure smooth limit functions (Fig. 3 (b)). We need more, namely, continuity of derivatives. The sequences converging to the first derivatives of the $\varphi^{s}(t)$ are the first-order finite differences

$$
\Delta g_{n}^{j, s}=\left(g_{n}^{j, s}-g_{n-1}^{j, s}\right) /(p / q)^{-j},
$$

which represent the slopes of the discrete curves $g_{n}^{3, s}$, plotted against $n(p / q)^{-j}$.

A remarkable fact is that derivatives can be obtained as limit functions of another rational filter bank, in which one factor $\frac{1-z^{-p}}{1-z^{-1}}$ in $G(z)$ has been replaced by $\frac{1-z^{-q}}{1-z^{-1}}$. This is a simple way to produce derivatives of rational wavelets.

To obtain $N$ th order continuous derivatives, one just has to consider $N$ th-order finite differences $\Delta^{N} g_{n}^{j, s}$ by applying $N$ times the operator $\Delta$ in (2), and impose that they converge uniformly. A necessary condition [1] is then that $G(z)$ should contain at least $N+1$ factors $\frac{1-z^{-p}}{1-z^{-1}}$, i.e.,

$$
\left(\frac{1-z^{-p}}{1-z^{-1}}\right)^{N+1} \text { divides } G(z)
$$


This gives a simple rule for constructing regular rational filter banks, already applied in [6] as a natural extension to the dyadic case. Adding one factor $\left(\left(1-z^{-p}\right) /\left(1-z^{-1}\right)\right)$, i.e., adding zeros at the $p$ aliasing frequencies $2 k \pi / p, 0 \leq k<p$, will generally increase the regularity order.

However, condition (3) is, mathematically speaking, not sufficient to obtain regularity: While zeros of the low-pass filter at the $p$ aliasing frequencies have a favorable effect for regularity, the effect of the other zeroes in $G(z)$ is generally destructive. Therefore, to quantify regularity accurately, we evaluate precisely the amount of regularity lost by these zeroes. To do this, we use the Hölder definition of regularity $[4,7,8]$ which allows us to extend the definition of regularity orders to real-valued numbers: The $\varphi^{s}(t)$ 's are said to be regular of order $N+\alpha$, where $0<\alpha \leq 1$, if there is a constant $c$ such that the $N$ th derivatives of $\varphi^{s}(t)$, denoted by $\varphi_{s}^{(N)}(t)$, satisfy

$$
\left|\varphi_{s}^{(N)}(t+h)-\varphi_{s}^{(N)}(t)\right| \leq c|h|^{\alpha}
$$

for all $t$ and arbitrarily small $h$. In other words, infinitesimal slopes are allowed to increase indefinitely, but not faster than $|h|^{-(1-\alpha)}$.

We can prove that it is sufficient, in order to obtain regularity order $N+\alpha$ for all limit functions $\varphi^{s}(t)$, that the following discrete rewriting of (4),

$$
\left|\Delta^{N} g_{n+1}^{j, s}-\Delta^{N} g_{n}^{j, s}\right| \leq c\left(\frac{p}{q}\right)^{-j \alpha}
$$

is satisfied. Now, to estimate (5), we extend the remark made earlier for derivatives to higher orders: The $N$ th derivative of $\varphi^{s}(t)$ is obtained by replacing $N$ factors $\frac{1-z^{-p}}{1-z^{-1}}$ in $G(z)$ by factors $\frac{1-z^{-q}}{1-z^{-1}}$. If we make still another replacement of this kind-assuming (3)-in order to produce the desired sequence in the left-hand side of (5), we obtain

$$
F_{N}(z)=\left(\frac{p}{q}\right)^{N}\left(\frac{1-z^{-q}}{1-z^{-p}}\right)^{N+1} G(z) .
$$

(The constant factor is there for normalization purposes.) Iterating $F_{N}(z)$ similarly as in (1) to give $F_{N}^{j}(z)$, is easy to check that the l.h.s. of (5) is the output of the flow-graph depicted in Fig. 2, in which $G^{j}(z)$ has been replaced by $F_{N}^{j}(z)$, and where the input is now the sequence associated to $z^{-s}\left(1-z^{-1}\right)^{N+1}$. Because of this latter property, it is now easy to estimate $\alpha$ in (5) using bounds on the coefficients of $F_{N}^{j}(z)$. We then obtain $r=N+\alpha$ as a sharp estimate of the regularity order.

We have deliberately left out many details, which are explained in the dyadic case in [8], and more or less easily carry over to the rational case.

\section{REGULARITY ESTIMATES}

We have seen in the preceding section that sharp regularity estimates are obtained simply by estimating $\alpha$ in (5), and that this is done using bounds on $F_{N}^{j}(z)$, an iterated version of (6). We now propose two algorithms to effectively compute $\alpha$. They differ only by the way bounds on $F_{N}^{J}(z)$ are computed.

\subsection{ITERATED ESTIMATES}

The following algorithm sharply estimates the regularity order, for any filter bank: First, normalize $G(z)$ such that $G(z=1)=p$ and consider $F_{N}(z)(6)$, in which zeroes at aliasing frequencies no longer appear. Next, compute $F_{N}^{j}(z)$ (associated to sequence $\left.\left(f_{N}^{j}\right)_{n}\right)$ using iteration (1). The Hölder regularity estimate $N+\alpha_{j}$, improved as $j$ is taken larger, is given by

$$
\left(\frac{p}{q}\right)^{-j \alpha_{j}}=\max _{n} \sum_{k}\left|\left(f_{N}^{j}\right)_{n-p^{j} k}\right|
$$

This algorithm requires a finite number of steps and can be easily implemented. However, it requires more computation for a given filter length than in the dyadic case $[7,8]$ : Complexity grows as $q^{j}$ times the lengths of the sequences $g_{n}^{j, s}$, and $q=1$ in the dyadic case. A sharp estimate is nonetheless obtained fairly rapidly using (7). As an example, the first values of regularity estimates for Kovačević and Vetterli's choice [6], $G(z)=\left(1-z^{-p}\right) /\left(1-z^{-1}\right)^{3}$. $\left(1-z^{-q}\right) /\left(1-z^{-1}\right)^{3}, p / q=3 / 2$, are 2.924108, 2.929214, $2.932957,2.935751$ and 2.937873 for the first 5 iterations, while an upper bound, computed as shown below, is 2.9498 . The resulting "wavelets" are all almost three times continuously differentiable.

We remark, in passing, that due to the shift invariance satisfied in the dyadic case (section 1), these estimates turn out to be truely optimal for almost all possible "dyadic" wavelets $[8,7]$. In the more general, rational case, proving optimality is much more difficult, again because of lack of shift invariance. However the limit of $\alpha_{j}$ in (7) will give the best (largest) $\alpha$ in (5), and we conjecture that this corresponds to a sharp regularity estimate for the $\varphi^{s}(t)$ 's.

\subsection{MATRIX ESTIMATES}

A variation of the above algorithm uses matrices, and is a natural extension to the work of Daubechies and Lagarias [4] for rational filter banks. Although their approach, based on two-scale difference equations, can no longer be used directly in the rational case (still because of lack of shift invariance), our discrete-time approach allows an easy generalization.

The idea is to compute the coefficients of $F_{N}^{j}(z)$ as entries of a matrix product, each matrix representing an elementary sampling change operation in an analysis filter bank. We then obtain

$$
\left(\frac{p}{q}\right)^{-j \alpha_{j}}=\max _{r_{k}}\left\|\prod_{k=1}^{j} F_{r_{k}}\right\|_{1}
$$

where each $\mathbf{F}_{r_{k}}$ is taken in a set of $p$ matrices $F_{r}, r=0$, $\ldots, p-1$. The latter are obtained as convolution matrices in which one have retained only one every $p$ th line and one every $q$ th column. (That is, $F_{r}$ has $(i, j)$ entries given by $\left.\left(f_{N}^{j}\right)_{r+i p-j q .}\right)$

The main advantage of this matrix formulation is that it provides sharp lower and upper bounds for regularity estimates provided by (7). A lower bound is obtained by replacing the right-hand side in (8) by the maximum of the 
Fr's norms (for any matrix norm), while an upper bound is obtained by taking spectral radii (maximum absolute value of eigenvalues) instead.

In contrast with (7), such bounds can be easily and efficiently computed. For the Kovačević and Vetterli's example considered above, we obtain $2.9241 \leq r \leq 2.9498$. In this case, the lower bound is precisely the first estimate $2+\alpha_{1}$ for $j=1$ in (7).

\section{SHIFT INVARIANCE}

As pointed out above, the major difficulty of the rational case as compared to the dyadic case is the lack of shift invariance of the equivalent impulse responses, resulting in a infinite collection of possible wavelet shapes rather than one. In other words, we obtain only a pseudo-wavelet transform, in which wavelet bases are not shifted versions of each other at a given scale. However, we now show that this shift invariance can be obtained approximately by using regular filters, as was observed in Fig. 3 (a). We prove the following result: if $N$ in (3) increases indefinitely, thereby increasing the regularity order $N+\alpha$, then the pointwise difference between two limit functions $\varphi^{s}(t+s)$ and $\varphi^{s^{\prime}}\left(t+s^{\prime}\right)$ tends to zero. The proof is a little intricate, and is outlined below.

To simplify the following derivations, we consider an average limit function, $\tilde{\varphi}(t)$, defined in [1] as

$$
\tilde{\varphi}(t)=\lim _{S \rightarrow \infty} \frac{1}{2 S+1} \sum_{|s| \leq S} \varphi^{s}(t+s)
$$

The problem amounts to finding an upper bound for the shift error

$$
\varepsilon=\sup _{t, s}\left|\varphi^{s}(t)-\tilde{\varphi}(t-s)\right|
$$

We found that, provided that the regularity order is positive, a bound for $\varepsilon$ is

$$
\varepsilon \leq c \sup _{t, s}\left|\tilde{\varphi}(t-s)-\sum_{n \in \mathbf{Z}} g_{q n-p s} \tilde{\varphi}((p / q) t-n)\right|
$$

Now, we recognize a two-scale difference equation $[1,4]$ in the term inside bars: Had shift invariance be statisfied, this term would vanish. However, because of the averaging procedure in (9), $\tilde{\varphi}(t)$ satisfies another two-scale difference equation, namely $\tilde{\varphi}(t-s)=\sum_{n} g_{q n-p s} \tilde{\varphi}((p / q) t-n)$, where $n$ is now taken over values $k / q$, where $k$ is an integer. Therefore, the sum in (10) reduces to non-integer values of $n$. It is now easy (?), taking Fourier transforms and using (3), to bound $(10)$ by the integral

$$
\varepsilon \leq c \max _{1 \leq k<q} \int|\operatorname{sinc}(\nu) \operatorname{sinp}((k+\nu) / q)|^{N} d \nu
$$

where we have noted $\operatorname{sinc}(t)=\sin (\pi t) /(\pi t)$ and $\operatorname{sinp}(t)=$ $\sin (\pi p t) / \sin (\pi t)$. Because $k$ is not a multiplie of $q$, this integral tends to zero exponentially as $N$ increases. Therefore, shift invariance is obtained within a small error as regularity increases.

We have seen that Kovačević and Vetterli's pseudowavelets, derived from their example in [6], are all almost three times continuously differentiable. Hence, they produce a very small shift error (We found $\varepsilon<10^{-2}$ ). This is the reason why these authors first thought that they obtained regular wavelets by plotting $g_{n}^{j}$ instead of $g_{n}^{j, s}:$ the curves of $\varphi^{s}(t)$ almost coincide within less than $10^{-2}$ error, similarly as in Fig. 3 (a).

\section{CONCLUSION}

Regularity requires smooth time evolutions of equivalent impulse responses in a rational filter bank. We believe that this property may be relevant for applications such as image coding for the same reasons as in the dyadic case [10]. As in the dyadic case, whether regularity is actually useful in a particular application remains an open question. The regularity estimates derived in this paper can be used as tools for investigating the precise role of regularity.

Even if the usefulness of smooth impulse responses is still an open question in coding applications, regularity has an additional merit in the rational case: it allows one to obtain shift invariance of rational filter banks, within a small error. In fact, lack of shift invariance may be a serious drawback, since the transform coefficients depend not only on the signal waveform, but also on the location at which it is analyzed-equivalent impulse responses vary in time. Also, efficient implementation of a true continuous-time wavelet transform with rational sampling changes can be obtained within a small error, based on the pseudo-shift invariance property. This is because shift invariance is necessary to utilize filter bank schemes using identical building blocks repeatedly. Techniques similar to what was done in the dyadic case [9] can then be applied in the rational case.

\section{REFERENCES}

[1] T. Blu, "Iterated filter banks with rational factors. Links with discrete wavelet transforms," IEEE Trans. Signal Processing, 1992. Submitted.

[2] A. Cohen and I. Daubechíes, "Non-separable bidimensional wavelet bases," Revista Matematica Iberoamericana, 1992. To appear.

[3] I. Daubechies, "Orthonormal bases of compactly supported wavelets," Comm. Pure Applied Math., vol. XLI, no. 7, pp. 909-996, 1988.

[4] I. Daubechies and J. Lagarias, "Two-scale difference equar tions II. Local regularity, infinite products of matrices and fractals," SIA M J.Math. Anal., 1990. To appear.

[5] J. Kovačević, Filter Banks and Wavelets: Extensions and Applications, $\mathrm{PhD}$ thesis, Columbia University, New York, NY, USA, 1991.

[6] J. Kovačević and M. Vetterli, "Perfect reconstruction filter banks with rational sampling rate changes," in Proc. ICA SSP, May 1991, pp. 1785-1788.

[7] O. Rioul, "A simple, optimal regularity estimate for wavelets," in Proc. European Signal Processing Conf. (EU. SIPCO), (Brussels), pp. 937-940, 1992.

[8] O. Rioul, "Simple regularity criteria for subdivision schemes," SIAM J. Math. Anal., vol. 23, no. 6, pp. 1544-1576, Nov. 1992.

[9] O. Rioul and P. Duhamel, "Fast algorithms for discrete and continuous wavelet transforms," IEEE Trans. Inform. Theory, vol. 38, no. 2, Mar. 1992.

[10] O. Rioul and M. Vetterli, "Wavelets and signal processing," IEEE Signal Processing Magazine, vol. 8, no. 4, pp. 14-38, Oct. 1991. 